\title{
Vitamin B12 Deficiency among Boarding School Children from Seven Districts of Bhutan
}

\author{
Laigden Dzed ${ }^{1}$, Hari Prasad Pokhrel ${ }^{* 2}$, Loday Zangpo ${ }^{1}$, Dorji Pelzom ${ }^{3}$, Ugyen Dendup ${ }^{1}$ \\ ${ }^{1}$ Department of Public Health, Ministry of Health, Bhutan \\ ${ }^{2}$ Gidakom Hospital, Ministry of Health, Bhutan \\ ${ }^{3}$ Policy and Planning Division, Ministry of Health, Bhutan
}

*Corresponding author: Hari Prasad Pokhrel; hari88pokhrel@ gmail.com

Received 02 August 2019;

Accepted 13 August 2019;

Published 29 August 2019

\begin{abstract}
Introduction: Bhutanese school children are vulnerable to vitamin B12 deficiency as outbreaks of micronutrient deficiency diseases have been a common occurrence. The study presents the status of vitamin B12 deficiency among boarding school children from those seven districts. Methods: A cross-sectional study was conducted to determine serum vitamin B12 level. Data and blood samples were collected from 448 boarding school children from the seven districts of Bhutan. Serum cobalamin levels were assessed and relationship between factors analyzed. Results: The study found that $64 \%$ of the school children were found to have vitamin B12 deficiency. Adjusted Odds Ratio for the vitamin deficiency among boarding school children from lower secondary and higher secondary schools were 4.05 and 3.3 respectively, when compared to those from the primary school. Starches were the most commonly served foods in boarding schools, while the animal source foods were served twice or less in a month. Conclusion: The study found a high prevalence of Vitamin B12 deficiency among boarding school children from seven districts. Boarding school meals had very less frequency of animal source foods.
\end{abstract}

Keywords: Vitamin B12 deficiency, Micronutrients, Animal source foods, School children, Bhutan

\section{Introduction}

Vitamin B12 or cobalamin in humans play an important role in the DNA synthesis ${ }^{[1]}$, and its deficiency is known to cause many hematologic, neurologic, and psychiatric impairments ${ }^{[1,2]}$. The vitamin is sometimes produced by micro-organisms in the large bowel of human intestine but is not absorbed, thus humans solely depend on dietary intake ${ }^{[3]}$. The major sources of vitamin B12 are animal source foods, mainly meats, eggs and dairy products ${ }^{[3]}$.

The two main causes of vitamin B12 deficiency are inadequate dietary intake and malabsorption ${ }^{[4]}$. In the developed countries malabsorption is the most common cause of vitamin B12 deficiency as the diet is rich in meat and other foods rich in vitamin B12. Most people with an insufficient vitamin B12 intake are, however, poverty-imposed vegetarians living in developing countries and represent a worldwide health problem ${ }^{[5]}$. Strict vegetarians are at even higher risk of vitamin B12 deficiency ${ }^{[6]}$.

High prevalence of vitamin B12 deficiency have been seen in the populations of Nepal, India and Bangladesh ${ }^{[7-9]}$. A recent study from India reported that $32 \%$ of the healthy school children are deficient in vitamin B12 which was even more pronounced in the rural areas and in school children who had poor dietary intakes ${ }^{[10]}$. Bhutanese school children are also vulnerable to the deficiency of this vitamin as outbreaks of micronutrient deficiency diseases have been a common occurrence ${ }^{[11,12]}$. The causes of these outbreaks in the Bhutanese school children have been attributed to poor dietary intakes of micronutrient rich foods, including animal source foods ${ }^{[11]}$. Therefore, it is very plausible that vitamin B12 could be very much deficient among the school children of Bhutan.

In 2014, a study to assess the status of thiamin deficiency among boarding school children was conducted in seven districts of Bhutan that had previous history of micronutrient deficiency disease outbreaks ${ }^{[11]}$. During the study, serum vitamin B12 was also measured, and therefore this study presents the status of vitamin B12 deficiency among boarding school children from those seven districts.

\section{Methods}

\section{Sample size estimation and sampling design}

The thiamine study ${ }^{[11]}$ was a prospective study to examine the status of thiamine deficiency amongst the boarding school children through the school academic year. The data for the thiamine study was collected in four phases and the data for this study was collected in November 2014 which coincided with the fourth phase of the thiamine study. The permission to use the dataset of the 
thiamine study for the analysis of this study was received from the principle investigator of the previous study.

The sample size was designed to produce statistically reliable estimates from the seven districts of Chukha, Lhuntse, Mongar, Pema Gatshel, Samdrup Jongkhar, Trongsa and Zhemgang.

Deciding on the Confidence Interval $(\mathrm{CI})$ of $95 \%(\mathrm{Z} \alpha=1.96)$ and the margin of error $(\epsilon)$ at 0.05 ; the sample size (n) was calculated using the sample size formula for the predetermined CI and $\epsilon$.

$$
n=\left[Z_{\alpha}^{2} * \sigma(1-\sigma)\right] / \epsilon^{2}
$$

Since there was no previous reference for variance, the standard deviation ( ) of 0.5 was set to yield the optimum number of subjects for the study. Using this formula and accounting for some dropout rates the final sample size was 448 .

Using probability proportional to size (PPS), 19 schools were selected from the seven districts and a minimum of 20 school children aged 9-22 years were selected from each school by simple random sampling. Ethical approval was granted by the Research Ethics Board of Health, Ministry of Health, Bhutan and administrative approval by the Ministry of Education. The study methodology is described in detail elsewhere ${ }^{[11]}$.
Data, sample collection and management

Blood samples were drawn following an overnight fasting from the subjects which were then transported to Public Health Laboratory (now known as Royal Center for Disease Control) based at Thimphu. Dietary information of the participants was obtained using a 43 item food frequency questionnaire (FFQ) and the subjects personal and family information were used from the thiamine study obtained during the first study period.

Cobalamin levels were estimated in the serum of the collected blood samples by Roche Elecsys modular Cobas e 411 using electrochemiluminescence immuno assay (Manheim Germany) using commercially available kits from Roche. The biochemical cobalamin deficiency was defined at a concentration below 200 $\mathrm{pg} / \mathrm{ml}$.

Data was entered in Epi-Data software version 3.1 and transferred to $\mathrm{R}$ version 3.1.1 and STATA version SE 12. Descriptive statistics were obtained for all variables. The odds ratios (OR) were adjusted for sex, class of student and education level and employment type of the child's supporter. Adjusted OR and confidence intervals assessed the relationship between above mentioned factors for the deficiency status.

\section{Results}

Table 1: Distribution of participants by Socio Demographic Characteristics $(n=448)$

\begin{tabular}{|c|c|c|c|}
\hline Characteristics & Frequency & $\%$ Mean & \\
\hline \multicolumn{4}{|l|}{ Gender } \\
\hline Male & 249 & $55.6 \%$ & \\
\hline Female & 199 & $44.4 \%$ & \\
\hline Age (mean) & 448 & 15.9 & $(\mathrm{SD}=2.684)$ \\
\hline Male & 249 & 16.1 & $(\mathrm{SD}=2.836)$ \\
\hline Female & 149 & 15.6 & $(\mathrm{SD}=2.462)$ \\
\hline \multicolumn{4}{|l|}{ Schooling Level } \\
\hline Primary & 110 & $24.6 \%$ & \\
\hline Lower secondary & 92 & $20.5 \%$ & \\
\hline Middle secondary & 149 & $33.3 \%$ & \\
\hline Higher Secondary & 97 & $21.7 \%$ & \\
\hline \multicolumn{4}{|l|}{ Care giver of the child } \\
\hline Both parents & 353 & $78.8 \%$ & \\
\hline Single parent & 80 & $17.9 \%$ & \\
\hline Others & 15 & $3.4 \%$ & \\
\hline \multicolumn{4}{|l|}{ Education of care giver } \\
\hline None & 252 & $56.3 \%$ & \\
\hline Non-formal/Religious & 96 & $21.4 \%$ & \\
\hline Primary and Less & 65 & $14.5 \%$ & \\
\hline Secondary and Above & 35 & $7.8 \%$ & \\
\hline \multicolumn{4}{|l|}{ Employment of the care giver } \\
\hline Salaried & 53 & $11.8 \%$ & \\
\hline Self-employed & 153 & $34.2 \%$ & \\
\hline Non-salaried & 242 & $54 \%$ & \\
\hline
\end{tabular}

\section{Socio-Demographic Characteristics}

Table 1 shows the distribution of participants by sociodemographic characteristics; a total of 448 school children participated in the study of which $44.4 \%$ were female. The average mean of the participants was around 16 years. Majority of the participants received care and support from both parents (care/support for children's learning in terms of monetary support and involvement in the children's development). Very few participants reported the major source of the income of their supporter from regular salaried wages. 
Table 2: Status of Vitamin B 12 among the boarding school children

\begin{tabular}{|c|c|c|c|}
\hline Characteristics & $\mathbf{n}$ & $\% *$ & $95 \% \mathrm{CI}$ \\
\hline \multicolumn{4}{|l|}{ Percentage deficiency } \\
\hline Male & 237 & 64.9 & $64.0-65.8$ \\
\hline Female & 190 & 64.4 & $63.5-65.4$ \\
\hline Total & 427 & 64.7 & $64.1-65.4$ \\
\hline Blood serum Vitamin B12 level & $\mathbf{n}$ & Median (pg/ml) & IQR pg/ml) \\
\hline Male & 237 & 149.9 & 116.7 \\
\hline Female & 190 & 154.7 & 118 \\
\hline Total & 427 & 150.8 & 117.6 \\
\hline
\end{tabular}

*weighted percentage

Prevalence of vitamin B12 deficiency

The prevalence of vitamin B12 deficiency among boarding school children is presented in table 2. Over half of the participants (64\%) were found to be deficient in vitamin B12. The median level of Vitamin B 12 was $150.8 \mathrm{pg} / \mathrm{ml}$.

Table 3: Socio-demographic factors associated with vitamin B 12 deficiency

\begin{tabular}{|c|c|c|c|c|c|}
\hline & & \multicolumn{2}{|c|}{ Unadjusted } & \multicolumn{2}{|c|}{ Adjusted $^{\wedge}$} \\
\hline \multicolumn{2}{|l|}{ Characteristics } & OR & $95 \% \mathrm{CI}$ & OR & $95 \% \mathrm{CI}$ \\
\hline \multicolumn{6}{|l|}{ Gender } \\
\hline & Male (Ref) & & & & \\
\hline & Female & 0.92 & $0.61-1.38$ & 0.93 & $0.59-1.45$ \\
\hline \multicolumn{6}{|c|}{ Participant's school level } \\
\hline & \multicolumn{5}{|l|}{ Primary (Ref) } \\
\hline & Lower secondary & $5.94 *$ & $3.11-11.35$ & $4.05 *$ & $1.87-8.76$ \\
\hline & Middle secondary & $5.76^{*}$ & $3.29-10.08$ & $3.3^{*}$ & $1.40-7.82$ \\
\hline & Higher Secondary & $4.78 *$ & $2.60-8.80$ & 2.58 & $0.87-7.62$ \\
\hline \multicolumn{6}{|c|}{ Education level of care giver } \\
\hline & \multicolumn{5}{|l|}{ None (Ref) } \\
\hline & Non-formal/Religious & 0.55 & $0.33-0.90$ & 0.77 & $0.44-1.34$ \\
\hline & Some form of formal Education & 0.69 & $0.41-1.14$ & 0.88 & $0.49-1.59$ \\
\hline \multicolumn{6}{|c|}{ Employment of care giver } \\
\hline \multicolumn{6}{|c|}{ Salaried/Self-employed (Ref) } \\
\hline & Non-salaried & 0.74 & $0.49-1.12$ & 0.8 & $0.50-1.29$ \\
\hline \multicolumn{6}{|l|}{ Care giver type } \\
\hline & Both Parents (Ref) & & & & \\
\hline & Single Parents & $1.54 *$ & $0.86-2.75$ & 1.47 & $0.78-2.77$ \\
\hline
\end{tabular}

* significant at p-value 0.05

$\wedge$ Adjusted for gender, schooling level, education level of the care giver, employment status of care giver and type of care giver

Socio-demographic factors associated with vitamin B12 deficiency

Table 3 shows the factors associated with vitamin B12 deficiency presented by both the crude and adjusted odds ratio. School children studying in lower secondary and middle secondary levels at the time of the study had 4 and 3 times higher odds respectively of having vitamin B12 deficiency when compared against that of the primary level. At higher secondary level, this difference disappears. 


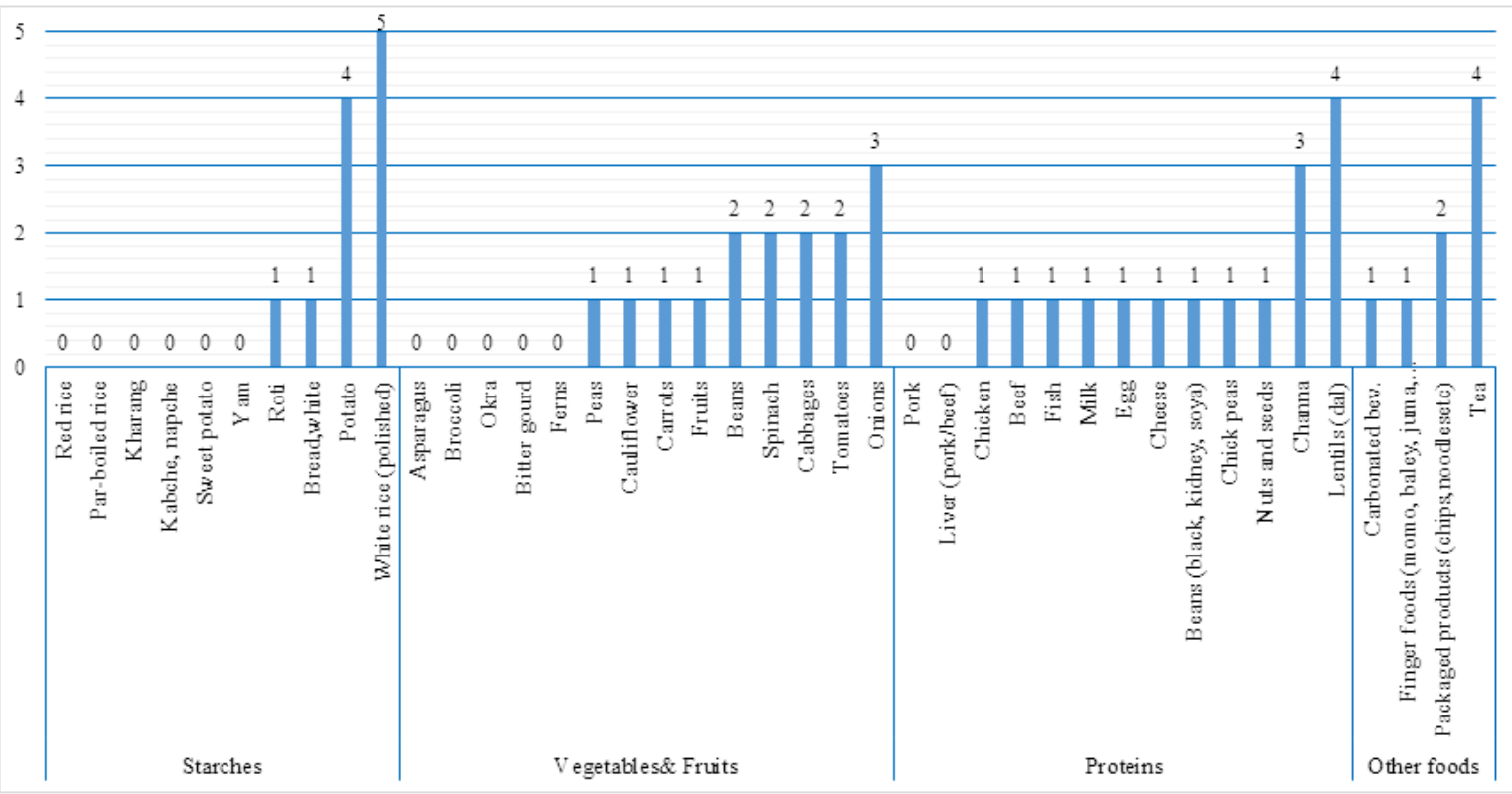

O:- Never; 1:-Twice per month or less; 2:- Once or twice per week; 3:- Three to six times per week; 4:-Once per day; 5:-More than once per day

\section{Figure 1: Frequency of food intake in the past one month}

\section{Frequency of food intake in the past one month}

Figure 1 describes the frequency of foods consumed over a month preceding the date of data. The most commonly consumed starches were potatoes and polished rice, which were consumed on an average, at least once a day. The intake of vegetables and fruits were generally low. Animal source foods were either never consumed (pork and liver) or consumed less frequently (chicken, beef, fish, milk, egg, cheese). Lentils and chick peas were reported to be the major source of proteins for the participants. Other foods like carbonated drinks, finger foods and packaged foods were consumed less frequently while tea was consumed once every day.

\section{Discussion}

This study found that $64 \%$ of the boarding school children from seven districts of Bhutan were deficient in vitamin B12. This is double the rate observed among the Indian school children ${ }^{[10]}$. This suggests that Bhutanese school children are even more vulnerable to the vitamin deficiency when compared to the counterparts in the neighboring country. This study also found generally low intake frequency of all animal sourced foods. The Indian study reported of positive association of serum B12 with dietary vitamin B12 intake $^{[10]}$. However, the cause of the high rates of vitamin B12 deficiency among the school children may not entirely be due to suboptimal school feeding, as human body can maintain large stores of vitamin B12 that can last up to 2 to 5 years ${ }^{[13]}$. Deficiency is usually caused by insufficient intake of animal source foods over long periods of time ${ }^{[13]}$. The high prevalence of Vitamin B12 deficiency in the school children indicates that the vitamin B12 deficiency could be widespread in the general population of Bhutan. Evidences indicate that B12 deficiency is much more common than it is perceived ${ }^{[5]}$. A national level multiple micronutrient survey in Bhutan is needed to ascertain this.

The risk of Vitamin B12 deficiency is even more in vegetarians because of the suboptimal intake of the vitamin ${ }^{[14]}$. This is important as vegetarianism is increasingly becoming popular in the country ${ }^{[15]}$. This could have a public health bearing as this population will be at risk of many micronutrient deficiencies. Even if the school children are not vegetarians, this study found that animal source foods in the school meals were served with minimal frequency which makes them very vulnerable to the micronutrient deficiency. However, this was not very different from the practice in the general population as the nationwide nutrition survey of 2015 showed that animal sourced foods are the least consumed in Bhutanese households ${ }^{[16]}$. Further analysis of the national survey indicated that a lot of the determinants of malnutrition were due to improper feeding practices ${ }^{[17]}$.

A study conducted in 2014 among boarding school children reported of thiamine deficiency ${ }^{[11]}$. The study also found that dietary intake of most of the micronutrients did not meet the requirements of the school children ${ }^{[11]}$. Although schools may not be the major cause of the vitamin deficiency, they can be part of the solution to solve a lot of micronutrient deficiencies in Bhutan. In one school in Kenya, the high prevalence of cobalamin deficiency among the school children was significantly reduced after supplementing with animal source foods ${ }^{[18]}$.

There were two major limitations in this study. The study was conducted among the boarding school children who avail all their meals from school, so this study does not indicate the status of the vitamin among the day scholar students. This study was conducted in the seven districts of Bhutan that had previous history of peripheral neuropathy outbreaks, so the study cannot be generalized to the rest of the boarding schools in the country.

\section{Conclusion}

The study found high prevalence of Vitamin B12 deficiency among boarding school children from seven districts. Boarding school meals had very less frequency of animal source foods. The study recommends a national multiple micronutrient survey in Bhutan to ascertain the deficiency of important micronutrients among the general population.

\section{Acknowledgement}

The authors would like to thank Ministry of Education, Bhutan for granting the permission to conduct this study. The authors would also like to thank all the participants of this study. 


\section{Conflict of Interest}

The authors have no conflict of interest to declare

\section{Funding}

Funding was not required for the conduct of this study

\section{References}

[1] Gibson RS. Principles of nutritional assessment: Oxford university press, USA; 2005.

[2] Oh R, Brown DL. Vitamin B12 deficiency. Am Fam Physician. 2003;67(5):979-86.

[3] Watanabe F. Vitamin B12 sources and bioavailability. Experimental biology and medicine (Maywood, NJ). 2007;232(10):1266-74.

[4] Oh R, Brown DL. Vitamin B12 deficiency. Am Fam Physician. 2003;67(5):979-86.

[5] Stabler SP, Allen RH. Vitamin B12 deficiency as a worldwide problem. Annual review of nutrition. 2004;24:299-326.

[6] Pawlak R, Parrott SJ, Raj S, Cullum-Dugan D, Lucus D. How prevalent is vitamin $\mathrm{B}(12)$ deficiency among vegetarians? Nutrition reviews. 2013;71(2):110-7.

[7] Ahmed F, Prendiville N, Narayan A. Micronutrient deficiencies among children and women in Bangladesh: progress and challenges. Journal of Nutritional Science. 2016;5:e46.

[8] Ng'eno BN, Perrine CG, Whitehead RD, Subedi GR, Mebrahtu S, Dahal P, et al. High Prevalence of Vitamin B12 Deficiency and No Folate Deficiency in Young Children in Nepal. Nutrients. 2017;9(1):72.

[9] Sasidharan P. B12 deficiency in India. Archives of Medicine and Health Sciences. 2017;5(2):261-8.

[10] Chakraborty S, Chopra M, Mani K, Giri AK, Banerjee P, Sahni NS, et al. Prevalence of vitamin B12 deficiency in healthy Indian school-going adolescents from rural and urban localities and its relationship with various anthropometric indices: a cross-sectional study. Journal of human nutrition and dietetics : the official journal of the British Dietetic Association. 2018.

[11] Dzed L, Dorji T, Pelzom D, Dhakal GP, Yangchen P, Wangmo K. Status of Thiamin deficiency in boarding school children from seven districts in Bhutan with previous history of peripheral neuropathy outbreaks: a cohort study. Bhutan Health Journal. 2017;1(01):49-56.

[12] Tsheten, Laigden D, Sangay T, Deki T, Karma D, Zangmo, et al. Investigation of suspected peripheral neuropathy outbreak in Cechentsemo Central School, Thinleygang, Punakha. Public Health of Indonesia. 2016;2(3):118-24.

[13] Snow CF. Laboratory diagnosis of vitamin B12 and folate deficiency: a guide for the primary care physician. Archives of internal medicine. 1999;159(12):1289-98.

[14] Pawlak R, Parrott SJ, Raj S, Cullum-Dugan D, Lucus D. How prevalent is vitamin B12 deficiency among vegetarians? Nutrition reviews. 2013;71(2):110-7.

[15] Chophel D, Thinley S, Gyaltshen D. Animal Wellbeing. Buddhism without Borders. 2012.

[16] National Nutrition Survey (NNS). Thimphu: Nutrition Program, Department of Public Health, Ministry of Health; 2015.

[17] Determinants of stunting, wasting, anemia and Infant \& young child feeding (IYCF) in Bhutan: secondary analysis of the national datasets from the National Nutrition Survey 2015. Thimphu, Bhutan: Nutrition Program, Department of Public health, Ministry of Health; 2017.

[18] McLean ED, Allen LH, Neumann CG, Peerson JM, Siekmann JH, Murphy SP, et al. Low plasma vitamin B12 in Kenyan school children is highly prevalent and improved by supplemental animal source foods. The Journal of nutrition. 2007;137(3):676-82. 